\title{
Surgical management of coronary artery arising from the wrong coronary sinus, using standard and novel approaches
}

\author{
Rajeev Gulati, MD, ${ }^{a}$ Vadiyala Mohan Reddy, MD, ${ }^{\text {a }}$ Casey Culbertson, MD, ${ }^{\mathrm{b}}$ Gregory Helton, MD, ${ }^{\mathrm{b}}$ Sam Suleman, MD, \\ Olaf Reinhartz, MD, ${ }^{a}$ Norman Silverman, MD, ${ }^{\mathrm{c}}$ and Frank L. Hanley, MD ${ }^{\mathrm{a}}$
}

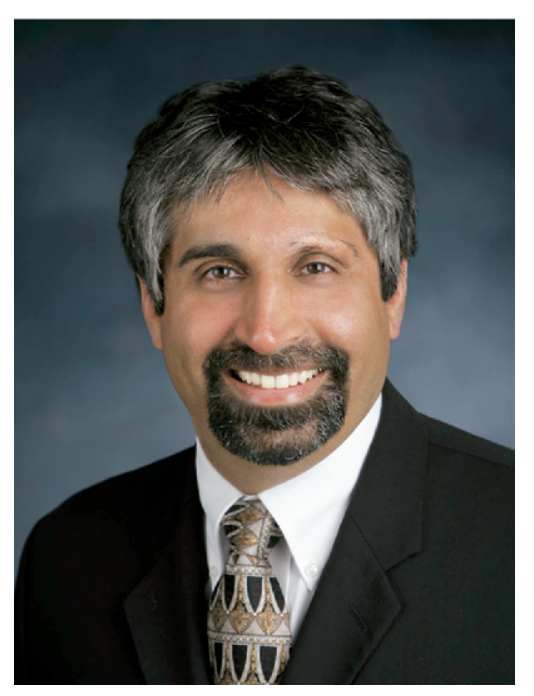

Dr Gulati

Supplemental material is available online.
From the Department of Pediatric Cardiac Surgery, Stanford University Medical Center $^{\mathrm{a}}$, Stanford, Calif; the Department of Pediatric Cardiology, Children's Hospital Oakland, ${ }^{\text {b }}$ Oakland, Calif; and the Department of Pediatric Cardiology, Stanford University Medical Center, ${ }^{c}$ Stanford, Calif.

Read at the Eighty-fifth Annual Meeting of The American Association for Thoracic Surgery, San Francisco, Calif, April 10-13, 2005

Received for publication June 22, 2006; revisions received Jan 29, 2007; accepted for publication Feb 14, 2007

Address for reprints: Rajeev Gulati, MD, Pomona Valley Hospital, 160 East Artesia Street, Suite 355, Pomona, CA 91767 (Email: rgulati@alumni.duke.edu).

J Thorac Cardiovasc Surg 2007;134:1171-8 0022-5223/\$32.00

Copyright $\odot 2007$ by The American Association for Thoracic Surgery

doi:10.1016/j.jtcvs.2007.02.051
Objectives: Patients with a coronary artery arising from the wrong sinus are susceptible to ischemia and sudden death. Risk is higher when the artery courses interarterially — between the pulmonary artery and aorta — has an intramural course, or has an abnormal orifice. In single coronary ostium without intramural course, unroofing and coronary reimplantation are inappropriate, and coronary artery bypass grafting is suboptimal. For this variant, we have devised pulmonary artery translocation.

Methods: A retrospective review of 18 patients undergoing repair between January 1999 and March 2005 was performed. Mean age was 8.1 years (range 6 weeks-16 years). All anomalous arteries coursed interarterially. Ten patients had a right coronary artery from the left coronary sinus; 8 had a left coronary artery from the right sinus. Eleven had an intramural course, and 4 had a single coronary ostium without an intramural course. Ten (56\%) patients had symptoms: chest pain (9/10), syncope (3/10), or dyspnea (2/10). Repair was implemented by unroofing $(\mathrm{n}=11)$, reimplantation $(n=3)$, or pulmonary artery translocation (1 lateral, 3 anterior). All patients were followed up clinically and echocardiographically.

Results: At a mean of 2.2 years ( 2 weeks- 5 years), there was no mortality. Symptoms improved and function remained normal in all but 1 patient. He had sustained multiple infarcts in the anomalous artery's distribution and required transplantation despite repair.

Conclusions: Repair is indicated in all patients with coronary insufficiency and in asymptomatic patients with high-risk morphologic abnormalities. We recommend unroofing when an intramural component (or slit-like orifice) is present, reimplantation for separate ostia without an intramural course, and pulmonary artery translocation for single ostium without an intramural course. Coronary artery bypass grafting is thus avoided.

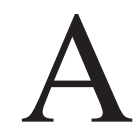
nomalous coronary artery arising from the wrong coronary sinus is a rare congenital anomaly; however, certain variants carry a high risk of mortality, with sudden death often being the first manifestation of disease. ${ }^{1-6}$ The morphologic spectrum of this entity encompasses the following: anomalous arteries with origin from any of the three aortic sinuses; interarterial course between the aorta and pulmonary artery (PA) versus noninterarterial course; intramural course in the wall of the aorta versus nonintramural course; single (common) ostium shared with the normal coronary artery versus separate ostium; and varying degrees of ostial compromise including slit-like ostium and angulation abnormalities. ${ }^{7,8}$

Large cohort studies indicate that death from these anomalies occurs only in patients who have an interarterial course or who have an abnormality of the coronary origin (such as a proximal intramural course, slit-like origin, or abnormally angulated origin). ${ }^{3,9-12}$ High-risk lesions include both anomalous right coronary 


\section{Abbreviations and Acronyms \\ LCA $=$ left coronary artery \\ LCS $=$ left coronary sinus \\ $\mathrm{PA}=$ pulmonary artery \\ $\mathrm{RCA}=$ right coronary artery \\ $\mathrm{RCS}=$ right coronary sinus}

artery (RCA) arising from the left coronary sinus (LCS) and anomalous left coronary artery (LCA) arising from the right coronary sinus (RCS) (Figure E1). Surgical correction is commonly recommended when one of these variants is identified, whether or not symptoms are present. It should be emphasized that coronary arteries that arise from the wrong coronary sinus and pass to the right of and posterior to the aorta, or to the left of and anterior to the PA, rather than passing between the PA and the aorta, are not believed to carry a high risk unless there is an obstructive lesion or abnormality of the coronary origin ${ }^{1,9,13}$ (Figure E2). Thus, these patterns do not warrant surgical intervention in the absence of coronary insufficiency.

Specific morphologic details influence the type of surgical repair. Mustafa and associates ${ }^{14}$ and Kaza, Tribble, and Crosby ${ }^{15}$ described "unroofing" of the intramural course of the coronary artery, which creates a new coronary ostium in the correct sinus, eliminates the course between the PA and aorta, and eliminates the intramural course (Figure E3).

When there are separate coronary ostia and no intramural course, coronary artery translocation and reimplantation have been used (Figure E4). Coronary artery translocation, however, is not advisable when the anomalous coronary artery shares a single ostium with the normal coronary artery, because it is difficult to obtain an adequate button and potentially compromises both coronary arteries.

In patients with a single coronary ostium and no intramural course, neither coronary artery translocation nor unroofing is an option. Coronary artery bypass grafting with the left internal thoracic artery or vein graft has been advocated by some, ${ }^{12,16}$ but there are a number of concerns associated with this approach. There is concern about involution as a result of competitive flow, because flow through the native coronary artery is normal most of the time and only becomes compromised intermittently with exercise. It has been suggested that the native artery be ligated to avoid competitive flow, but this creates an irreversible iatrogenic occlusion and makes the circulation completely dependent on a graft and anastomosis whose durability and growth potential are uncertain. ${ }^{12,17,18}$ These concerns are particularly applicable in children and adolescents, who comprise the majority of patients.

To address these concerns, Rodefeld and colleagues ${ }^{19}$ developed a new method of treatment, lateral PA translo- cation, which was described in 2002 (Figure E5). This technique and a new modification that we now describeanterior PA translocation (Figure 1) - were used in this series as part of a morphology-based management protocol. These techniques are described in the Patients and Methods section.

We have performed a retrospective review to assess the appropriateness of this morphology-based surgical management protocol, which incorporates both standard and novel treatments.

\section{Patients and Methods Study Design}

A retrospective analysis of all patients undergoing surgery for anomalous coronary artery arising from the wrong coronary sinus between January 1999 and April 2005 was performed. Surgery was performed at 3 hospitals by 5 surgeons in a unified program. Follow-up was by direct contact with patient families and primary cardiologists, and full access to all postprocedure studies was obtained. Follow-up was complete for all patients.

\section{Patient Characteristics}

Eighteen patients underwent repair. Patient characteristics are outlined in Table 1. There was a male predominance. RCA arising from the LCS and LCA arising from the RCS were approximately equally represented. The anomalous artery had an interarterial course in all 18 patients, an intramural course in 12, and a single coronary ostium in 5 . One patient with a single coronary ostium also had an intramural course, but none of these patients had an abnormality of the origin. Thus, there were 4 patients with single coronary ostium, no intramural course, and no abnormality of the coronary origin.

Most (13) patients had isolated anomalies, but 5 had minor associated anomalies that would not by themselves have justified intervention. One patient had a ventricular septal defect, but this was restrictive and the patient was free of symptoms. One patient had a bicuspid aortic valve without significant insufficiency or stenosis that was repaired, and 1 patient had a small coronarycameral fistula that was coil-occluded preoperatively.

Ten patients were symptomatic (Table 2). Three patients had sustained myocardial infarctions preoperatively, and 1 had experienced severe exercise-induced pulmonary edema.

Both of the patients with syncope had an anomalous LCA arising from the RCS. Two of the 4 patients with a single ostium and no abnormality of the coronary origin were symptomatic, 1 having exertional angina and myocardial infarction and the other having near syncope with exertion.

\section{Procedures and Technique}

Procedures performed are outlined in Table 3. All patients underwent operation via a median sternotomy, with moderate hypothermic cardiopulmonary arrest and cardioplegia-induced cardiac arrest.

Unroofing was performed whenever the anomalous coronary had an intramural course. The exact technique of repair was dependent on whether or not this course was superior to the commisures. If there was an intramural course and the course was close to or above the sinotubular junction, unroofing was per- 

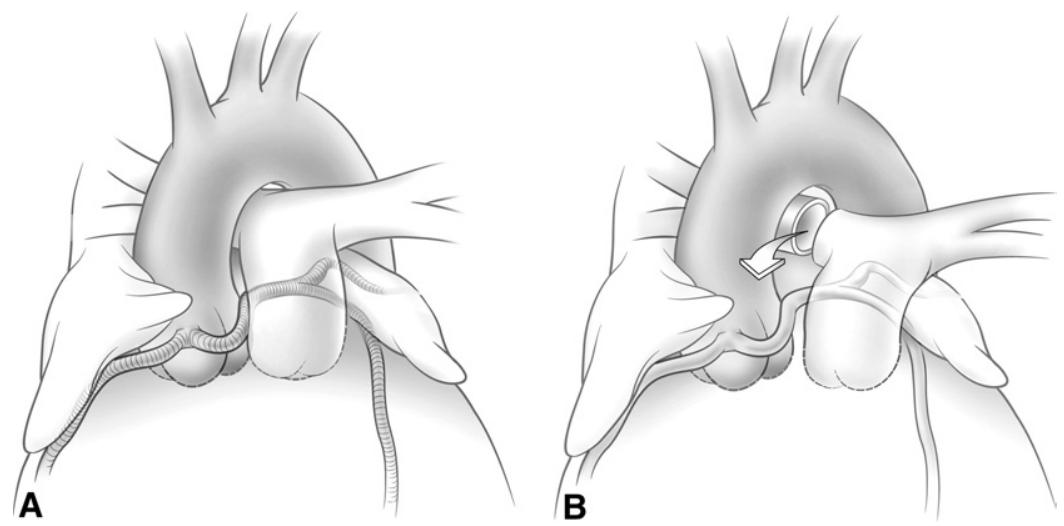

Figure 1. Anterior pulmonary artery (PA) translocation. A, Anomalous left coronary artery from the right coronary sinus (RCS), with single origin and normal proximal course. B, Both branch PAs are fully mobilized and right branch PA is transected and moved anterior to the aorta. C, The right PA is reattached and a pericardial patch is added, as necessary. This moves the main PA both anteriorly and leftward, relieving compression on the interarterial portion of the anomalous artery.

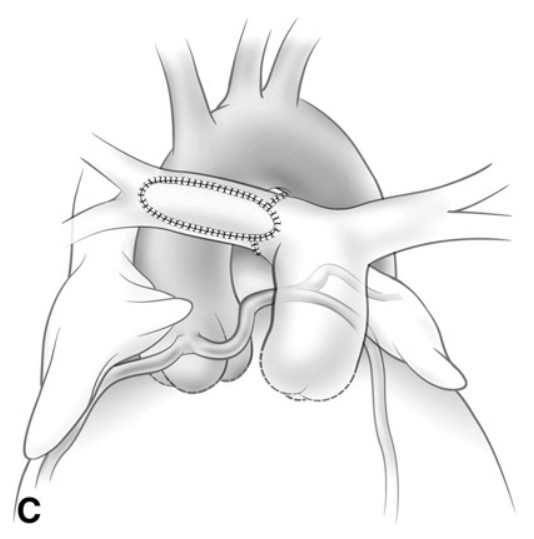

formed according to techniques described by Mustafa and associates ${ }^{14}$ and Kaza, Tribble, and Crosby ${ }^{15}$ (Figure E3). In some cases, it was necessary to detach the aortic commissure and resuspend it.

If the course was intramural and significantly below the sinotubular junction, a technique of limited unroofing or "fenestration" was used. This technique, used on 1 of the patients in this series, was previously reported by Karamichalis and coworkers. ${ }^{20}$ In this method, a small right-angle clamp or coronary probe is placed into the coronary ostium, passed into the intramural portion of the coronary artery, and used to define the course of the artery as it travels in the wall around the aortic commissure and enters the wall of the correct sinus. The portion of the course in the correct sinus is then unroofed or "fenestrated" with a number 11 blade and $2.5-\mathrm{mm}$ to 4.0-mm aortic punch (Figure E6). This avoids extensive takedown and resuspension of the aortic valve commissure, which can be associated with aortic insufficiency. ${ }^{21}$

If the course was not intramural and the coronary arteries had separate origins, the origin of the anomalous artery was excised as a button and reimplanted. The position of reimplantation was usually distal to the sinotubular junction, above the correct coronary sinus. This removed the coronary artery from between the aorta and PA, and the distal placement avoided kinking. The original site was closed with a patch of glutaraldehyde-treated pericardium (Figure E4).

If the course was not intramural and the coronary arteries had a single ostium, lateral or anterior PA translocation was performed. In lateral PA translocation, ${ }^{19}$ rather than trying to move the coronary artery out from between the aorta and PA, the PA is instead moved away from the coronary artery. This was designed to eliminate direct compression of the anomalous coronary artery between the two vessels and prevent exacerbation of angulation of the coronary origin during compression. The main PA was transected just before its bifurcation and reimplanted laterally on the left main PA. The original site was closed with a patch (Figure E5).

An additional variation, anterior PA translocation, which has not been previously described, was developed for situations in which anterior as well as left lateral displacement was judged to be potentially helpful. The main PA and its branches are fully mobilized, and then the right PA is transected and reconnected anterior to the ascending aorta. This moves the proximal main PA away from its contact point with the left anterolateral aspect of the aortic root (Figure 1).

\section{Preoperative and Postoperative Evaluation}

All patients had preoperative echocardiograms and coronary angiograms for diagnosis. Three patients had preoperative magnetic resonance imaging scans and 1 had a computed tomographic angiogram.

Of particular interest is 1 patient who had a single coronary ostium and LCA from the RCS without an intramural course or abnormality of the coronary origin. A preoperative echocardiogram clearly demonstrated dynamic compression of the interarterial course of the anomalous coronary artery, most prominent at end-systole to early-diastole. 
TABLE 1. Patient characteristics $(\mathbf{n}=\mathbf{1 8})$

\begin{tabular}{lc}
\hline Age & 6 mo-15 y \\
Range & $8 \mathrm{y}$ \\
Mean & \\
Sex & 13 \\
Male & 5 \\
Female & \\
Type of coronary anomaly & 8 \\
LCA from RCS & 10 \\
RCA from LCS & 18 \\
Interarterial course (between aorta and PA) & 12 \\
Intramural course & 5 \\
Single coronary ostium & 1 \\
$\quad$ Single coronary ostium, intramural course & 4 \\
$\quad$ Single coronary ostium, normal origin & 5 \\
Associated anomalies & 2 \\
$\quad$ PFO/ASD & 1 \\
Restrictive VSD and PFO & 1 \\
$\quad$ Bicuspid aortic valve & 1 \\
$\quad$ Coronary-cameral fistula & \\
\hline
\end{tabular}

$L C A$, Left coronary artery; $R C S$, right coronary sinus; $R C A$, right coronary artery; $L C S$, left coronary sinus; $P A$, pulmonary artery; $P F O$, patent foramen ovale; $A S D$, atrial septal defect; $V S D$, ventricular septal defect.

Postoperatively, patients were followed up clinically for symptoms, arrhythmia, and sudden death. All had electrocardiograms and echocardiograms for rhythm, function, and coronary anatomy. Four had stress tests and 2 had coronary angiograms.

\section{Results}

Mean follow up was 2.2 years (range 1 week-5 years). Two patients had mild postoperative pericarditis that resolved with medical treatment and 1 had transient complete heart block and had a pacemaker placed. Block had resolved by her 3-month follow-up and has not recurred. No sudden deaths occurred among these patients.

Symptoms improved and cardiac function remained normal in all but 1 patient. This 15 -year-old patient, since the age of 8 , had had 7 episodes of syncope and chest pain after running or playing football, each lasting up to an hour, and presented after one such episode with electrocardiographic changes consistent with anteroseptal myocardial infarction. An echocardiogram revealed poor left ventricular function and dilation as well as an anomalous coronary artery. He underwent a cardiac catheterization that confirmed anoma-

\section{TABLE 2. Patient presentation}

\begin{tabular}{lc}
\hline Symptomatic & 10 \\
Chest pain & 9 \\
Syncope or near syncope & 3 \\
Myocardial infarction & 3 \\
Dyspnea on exertion & 2 \\
Pulmonary edema & 1 \\
\hline
\end{tabular}

lous left main coronary artery arising from the RCS, with an intramural course, passing between the PA and aorta. Echocardiography confirmed compression. Preoperatively, he had junctional and supraventricular arrhythmias. After unroofing, despite a cardiac catheterization showing a totally normal LCA, his function remained poor with an ejection fraction in the $11 \%$ to $16 \%$ range, and arrhythmias continued. He had a pacemaker/defibrillator placed and was medically optimized, but heart failure worsened and he required placement of a left ventricular assist device. He subsequently underwent cardiac transplantation and is free of symptoms 18 months postoperatively. Examination of the explanted heart revealed multiple old infarctions in the distribution of the anomalous coronary artery. The coronary neo-ostium was patent.

At 1- to 2-year follow-up, 2 patients had had atypical, sharp chest pain, but echocardiograms, electrocardiograms, and coronary angiograms in 1 showed no abnormalities. The pain was diagnosed as noncardiac, probably musculoskeletal pain. Two other patients underwent stress tests, which showed no abnormalities.

Although our advice has been to continue to avoid strenuous athletics, 5 patients, all of whom were symptomatic preoperatively, have insisted on returning to sports. All are free of cardiac symptoms, although 1 has had musculoskeletal pain. One patient competes regularly in $800-\mathrm{m}$ to $1600-\mathrm{m}$ foot races, and another recently scored 16 goals on a water polo team ranked 17 th nationwide.

Among the 4 patients who underwent PA translocation, 2 had symptoms preoperatively. Postoperatively, all have normal cardiac function and electrocardiograms and none has cardiovascular symptoms. None has echocardiographic or angiographic evidence of coronary compression or PA stenosis. One had musculoskeletal chest pain, but echocardiogram, stress test, and angiography showed no abnormalities.

\section{Discussion}

Coronary artery arising from the wrong sinus encompasses a variety of specific morphologic lesions. It is widely documented that coronary insufficiency and sudden death are associated with some of these variants, specifically those that have a course that runs between the two great arteries and those that have abnormalities of the origin of the cor-

TABLE 3. Procedures performed $(\mathbf{n}=\mathbf{1 8})$

\begin{tabular}{lr}
\hline Unroofing or limited unroofing ("fenestration") & 11 \\
Unroofing & 8 \\
Fenestration & 3 \\
Coronary artery translocation and reimplantation & 3 \\
Pulmonary artery translocation & 4 \\
Lateral & 1 \\
Anterior & 3
\end{tabular}


onary artery. There is a degree of uncertainty regarding the exact pathophysiology underlying the observed morbidity, and this subject has been extensively discussed in the literature. . $^{71,16,17,22}$

Some skepticism remains regarding the role of compression of the anomalous coronary by the great vessels when the coronary has an interarterial course. However, both logic and some recent evidence support the importance of this pathophysiologic mechanism. First, a number of variants of coronary artery arising from the wrong sinus do not have a proximal course passing between the great arteries. These include anomalous coronaries arising from the RCS, LCS, and even noncoronary sinus. Coronary insufficiency is not associated with these variants, ${ }^{7,9,23}$ except when a concomitant obstructive coronary lesion, such as a slit-like coronary ostium, is also present. ${ }^{1,24}$ In contrast, sudden death has been reported and coronary insufficiency has been documented in several cases when an interarterial course is present with no other concomitant obstructive coronary lesions. ${ }^{1,10,12}$

In our own series, there were 2 symptomatic patients who had anomalous coronary artery from the wrong coronary sinus with single coronary ostium, no intramural course, and no abnormalities of the ostium. Compression between the great arteries was the only potential cause of obstruction. Both had resolution of symptoms after PA translocation, again suggesting that compression is etiologic. Furthermore, in a study attempting to identify risk factors for sudden death from anomalous coronary artery arising from the wrong coronary sinus and passing interarterially, comparing patients with the anomaly who suffered or did not suffer sudden death, no significant difference in the angle of coronary takeoff, the length of intramural segment, or the degree of displacement of the anomalous coronary artery from the correct coronary sinus was seen, which suggests that the important factor is the interarterial course itself. ${ }^{11}$ These observations underscore the importance of the interarterial factor as an etiologic agent in coronary insufficiency. In addition, improved imaging, including dynamic magnetic resonance imaging, ${ }^{25}$ multislice computed tomography, ${ }^{26}$ intravascular ultrasound, ${ }^{27}$ stress myocardial perfusion single photon emission computed tomography, ${ }^{28}$ and direct measurement of intracoronary pressure, ${ }^{29}$ has documented compression and inotrope-inducible pressure gradients within the segment of coronary artery between the great arteries.

When all of these factors are taken into consideration, a prudent management approach seems to be to operate on any variant associated with actual coronary insufficiency and all variants that pass between the great arteries, even if found incidentally.

Morphology also forms the basis of our surgical management protocol (Figure 2). The optimal operative proce-

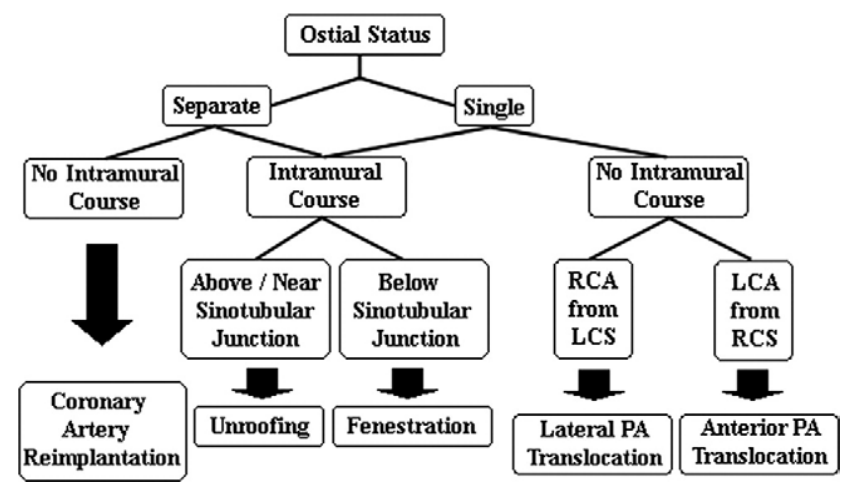

Figure 2. Morphology-based surgical management protocol. PA, Pulmonary artery; $R C A$, right coronary artery; $R C S$, right coronary sinus; $L C A$, left coronary artery; $L C S$, left coronary sinus.

dure is determined on the basis of the specific morphologic details of each lesion. Unroofing and translocation procedures are appropriate and effective for certain morphologic types, but not appropriate for all. Coronary artery bypass grafting has been advocated by some, especially for cases that are not appropriate for unroofing or translocation. ${ }^{12,16}$ We agree with those who believe that grafting is not the best solution, especially in children and adolescents, who comprise the majority of patients.

As an alternative, we have developed the PA translocation procedure, which is designed to convert patients from high-risk variants (those passing between the great arteries) to low-risk variants (those that do not pass between the great arteries), without actually moving the coronary artery itself. It is, of course, critical to document that other factors associated with coronary insufficiency, such as fixed obstructive coronary lesions or slit-like ostium, do not exist when this procedure is undertaken. Whether lateral or anterior translocation is chosen depends on the orientation of the coronary artery itself, and equally important, the specific positioning of the aortic and pulmonary roots. The point of potential compression ranges over a sector that is variable but always within the left anterolateral quadrant of the circumference of the aortic root. Depending on the intraoperative evaluation in each specific case, the more effective maneuver is chosen. Although the numbers are small, our impression is that LCA from the RCS is more likely to benefit from anterior PA translocation, and RCA from the LCS is more likely to benefit from lateral PA translocation.

Results of the study support the use of this protocol, although one must use caution when generalizing the results of a relatively small study with limited follow-up to all patients, particularly to those who have symptoms. Use of the various techniques, including the novel technique of PA translocation, carries low morbidity and mortality, maintains cardiac function, and improves symptoms in most 
cases, and it demonstrates freedom from sudden death at short- and midterm follow-up. Avoiding coronary artery bypass grafting is suggested, especially in young patients.

\section{References}

1. Taylor AJ, Rogan KM, Virmani R. Sudden cardiac death associated with isolated congenital coronary artery anomalies. J Am Coll Cardiol. 1992;20:640-7.

2. Maron BJ, Shirani J, Poliac LC, Mathenge R, Roberts WC, Mueller FO. Sudden death in young, competitive athletes: clinical, demographic, and pathological profiles. JAMA. 1996;267:199-204.

3. Corrado D, Thiene G, Nava A, Rossi L, Pennelli N. Sudden death in young competitive athletes: clinicopathologic correlations in 22 cases. Am J Med. 1990;89:588-96.

4. Zepilli P, dello Russo A, Santini C, Palmieri V, Natale L, Giordano A, et al. In vivo detection of coronary artery anomalies in asymptomatic athletes by echocardiographic screening. Chest. 1998;114:89-93.

5. Eckart RE, Scoville SL, Campbell CL, Shry EA, Stajduhar KC, Potter $\mathrm{RN}$, et al. Sudden death in young adults: a 25-year review of autopsies in military recruits. Ann Intern Med. 2004;141:829-34.

6. Ness MJ, McManus BM. Anomalous right coronary artery origin in otherwise unexplained infant death. Arch Pathol Lab Med. 1988;112: 626-9.

7. Felmeden D, Singh SP, Lip GY. Anomalous coronary arteries of aortic origin. Int J Clin Pract. 2000;54:390-4.

8. Davis JA, Cecchin F, Jones TK, Portman MA. Major coronary artery anomalies in a pediatric population: incidence and clinical importance. J Am Coll Cardiol. 2001;37:593-7.

9. Frescura C, Basso C, Gaetano T, Corrado D, Pennelli T, Angelini A, et al. Anomalous origin of coronary arteries and risk of sudden death: a study based on an autopsy population of congenital heart disease. Hum Pathol. 1998;29:689-95.

10. Moodie DS, Gill C, Loop FD, Sheldon WC. Anomalous left main coronary artery originating from the right sinus of Valsalva: pathophysiology, angiographic definition, and surgical approaches. J Thorac Cardiovasc Surg. 1980;80:198-205.

11. Taylor AJ, Byers JP, Cheitlin MD, Virmani R. Anomalous right or left coronary artery from the contralateral coronary sinus: "high-risk" abnormalities in the initial coronary artery course and heterogenous clinical outcomes. Am Heart J. 1997;133:428-35.

12. Duke C, Rosenthal E, Simpson JM. Myocardial infarction in infancy caused by compression of an anomalous left coronary artery arising from the right coronary artery. Cardiol Young. 2004;14:654-7.

13. De Luca L, Bovenzi F, Signore N, D’Agostino C, de Luca I. Primary angioplasty of an anomalous right coronary artery complicated by an acute thrombotic occlusion. Ital Heart J. 2004;5:785-8.

14. Mustafa I, Gula G, Radley-Smith R, Durrer S, Yacoub M. Anomalous origin of the left coronary artery from the anterior aortic sinus: a potential cause of sudden death. Anatomic characterization and surgical treatment. J Thorac Cardiovasc Surg. 1981;82:297-300.

15. Kaza AK, Tribble CG, Crosby IK. Repair of an anomalous left coronary artery. Cardiovasc Surg. 2002;10:276-8.

16. Cooley DA. Repair of coronary anomalies. In: Techniques in cardiac surgery. Philadelphia: WB Saunders; 1984. p. 264-1.

17. Beique F, Tran QH, Ma F, Rudski L, Daves S, Angelini P. Anomalous right coronary artery originating from the left sinus of valsalva. $J$ Cardiothorac Vasc Anaesth. 2004;18:788-98.

18. Pelliccia A. Congenital coronary artery anomalies in young patients. J Am Coll Cardiol. 2001;37:598-600

19. Rodefeld MD, Culbertson CB, Rosenfeld HM, Hanley FL, Thompson LD. Pulmonary artery translocation: a surgical option for complex anomalous coronary artery anatomy. Ann Thorac Surg. 2001;72: 2150-2.

20. Karamichalis JM, Vricella LA, Murphy DJ, Reitz BA. Simplified technique for correction of anomalous origin of left coronary artery from the anterior aortic sinus. Ann Thorac Surg. 2003;76:266-7.

21. Romp RL, Herlong JR, Landolfo CK, Sanders SP, Miller CE, Ungerleider RM, et al. Outcome of unroofing procedure for repair of anom- alous aortic origin of left or right coronary artery. Ann Thorac Surg. 2003;76:589-95; discussion 595-6.

22. Roberts WC, Siegel RJ, Zipes DP. Origin of the right coronary artery from the left sinus of Valsalva and its functional consequences: analysis of 10 necropsy patients. Am J Cardiol. 1982;49:863-8.

23. Basso C, Maron BJ, Corrado D, Thiene G. Clinical profile of congenital coronary artery anomalies with origin from the wrong aortic sinus leading to sudden death in young competitive athletes. $\mathrm{J}$ Am Coll Cardiol. 2000;35:1493-501.

24. Liberman L, Pass RH, Kaufman S, Hordof AJ, Printz BF, Prakash A. Left coronary artery arising from the non-coronary sinus: a rare congenital coronary anomaly. Pediatr Cardiol. 2005;26:672-4.

25. Bunce NH, Rahman SL, Keegan J, Gatehouse PD, Lorenz CH, Pennell DJ. Anomalous coronary arteries: anatomic and functional assessment by coronary and perfusion cardiovascular magnetic resonance in three sisters. J Cardiovasc Magn Reson. 2001;3:361-9.

26. Ghersin E, Litmanovich D, Ofer A, Lessick J, Dragu R, Rispler S, et al. Anomalous origin of right coronary artery: diagnosis and hemodynamic evaluation with multidectector computed tomography. J Comput Assist Tomogr. 2004;28:293-4.

27. Angelini P, Velasco JA, Flamm S. Coronary anomalies. Incidence, pathophysiology, and clinical relevance. Circulation. 2002;105:2449-54.

28. De Luca L, Bovenzi F, Rubini D, Niccoli-Asabella A, Rubini G, De Luca I. Stress-rest myocardial perfusion SPECT for functional assessment of coronary arteries with abnormal origin of course. J Nucl Med. 2004:45:532-6

29. Lim JM, Forsberg MJ, Lee R, Kern MJ. Hemodynamic abnormalities across an anomalous left main coronary artery assessment: evidence for a dynamic ostial obstruction. Catheter Cardiovasc Interv. 2004; 63:294-8.

\section{Discussion}

Dr James Jaggers (Durham, NC). In this paper the authors have reviewed their experience with these relatively rare and potentially lethal coronary artery anomalies. It is very difficult to know the actual prevalence of this lesion, but it is clear that as cardiologists' skill with echocardiography and increased suspicion of coronary artery lesions increases, we will be asked to render opinions regarding the indications and outcomes of surgical intervention. Studies like this add significantly to our understanding of the surgical options.

Our experience is similar to yours. We have previously reported on 9 patients and subsequently performed this operation on 12 other patients, with no morbidity or mortality. Our experiences suggest that repair can be accomplished in the vast majority of patients. The obvious question that remains unanswered is whether we are actually positively affecting the lifetime risk of these patients or are we creating new risk for the future.

Dr Gulati, you and your group have used relatively standard techniques of unroofing or modified unroofing or reimplantation in 14 patients. However, in 4 patients, in whom the LCA or the RCA arises from the contralateral sinus and travels between the PAs without taking an intramural course, you have proposed a separate operation that includes PA translocation. You postulate that this will effectively relieve compression of the coronary artery between the great vessels, presumably during exercise.

I have several questions for you.

You stated that all your patients had cardiac catheterization before surgery. Is this necessary, and what do you think is the optimal diagnostic tool to confirm this anatomy? The anatomy is very important, as you know, to determine whether it is an intramural course versus an extramural course.

Dr Gulati. All of our patients did receive cardiac catheterizations, because we thought that this was the best technique for 
diagnosis. However, some of the series in the literature have shown magnetic resonance imaging to be a very good tool. Computed tomographic scanning has also been used, but it is hard to use on smaller patients. Thus, I do not know that cardiac catheterization is necessarily the definitive tool. In fact, in a couple of our cases, there was a difference between the cardiac catheterization and the magnetic resonance or computed tomographic scan, and the scan was correct.

Dr Jaggers. In this group of patients that shared a common orifice with the RCA or the LCA, you chose to translocate the PAs. Were any of those 4 patients symptomatic before the operation?

Dr Gulati. Yes, 2 of those patients were symptomatic. One patient had chest pain and 1 had syncope. Both patients became free of symptoms after the operation.

Dr Jaggers. Some have recommended intracoronary stenting for this lesion in which the artery is not intramural. Do you have any experience or any recommendations regarding that?

Dr Gulati. We do not have any experience with stenting. Normally this diagnosis is made in children, and stenting is not necessarily a good option in children since they are growing. If you look at the literature, there was 1 case in which a left internal thoracic artery graft was done and a stent was eventually attempted because of stenosis. That patient died on the operating table. There is some good experience in the adult literature, so maybe it is reasonable to stent in an adult, but I would not recommend it in a child.

Dr Jaggers. For the PA translocations, as we know when we do circumferential PA anastomoses, we can either create functional or anatomic abnormalities of those PAs. Do you have any echocardiographic assurance that you have not created a problem with this type of technique?

Dr Gulati. Yes, I did carefully review the follow-up echocardiograms and there was no evidence of stenosis.

Dr Jaggers. I guess the difficult things to talk about are the appropriate age and indications to repair these abnormalities. What are your specific recommendations for the infant or neonate with anomalous aortic coronary artery, be it intramural or extramural? Specifically, would you repair any of these defects when they are discovered in preparation for another operation, for example, total anomalous pulmonary venous connection?

Dr Gulati. I have not encountered that situation, but I would recommend repair in a patient with both abnormalities.

Dr Jaggers. Finally, some of your patients were free of symptoms. I noticed that you commented that in the low-risk group you would not recommend operation. For what specific lesions would you not recommend operations? Would they include the anomalous RCA from the LCS with an intramural course?

Dr Gulati. In general, if you look in the pathologic series, the patients who had an intramural course were definitely at higher risk. In fact, in one series, $82 \%$ of the patients who had anomalous LCA from the RCS had sudden death from that abnormality. Risk was slightly lower for RCA from the LCS, but these are really among the high-risk lesions. Anybody with an interarterial course, anybody with an intramural course, and anybody with a slit-like coronary origin should undergo operation whether symptomatic or not.

Dr Jaggers. That has been our tactic as well. If you look at those series, very few of those patients died of sudden death before adolescence. We currently do not recommend elective repair in asymptomatic patients before that period of time.

Dr Thomas L. Spray (Philadelphia, $\mathrm{Pa}$ ). I think we are seeing more and more of these patients now as people are getting diagnostic imaging as a routine procedure for pre-sports-type interventions, for example. Thus symptomatic patients are now the rarity in our experience with these coronary anomalies. They are often being referred with very mild symptoms, if any, or some vague symptom that may or may not be related to their coronary disease. I think the techniques are fairly standard.

The subgroup you describe with the mobilization of the PA is an interesting and novel technique. What I am waiting to see from your institution is a real description of why this would work, especially with truly good imaging studies suggesting that you can move the proximal PA away from the aorta simply by moving the distal PA away from the distal aorta.

The pulmonary valve sits in the infundibulum. The annulus and the valve sit very close to the aortic annulus and to the coronary artery. Moving the PA distally over to the left may make a small difference proximally, but the amount of movement has to be relatively minimal since the annulus is fixed. I would encourage you to give us some images that show us that this actually does work and does relieve the compression. When you move the right PA anteriorly and then reattach it to the PA, you may also re-create an anterior compression of the PA and aorta. Although I think this is an interesting technique, and I have even done it on a couple of occasions, I really think that some good computed tomographic or magnetic resonance imaging would enhance the utility of this technique and give us better convincing data that it is actually moving the compression away from the coronary artery in these unusual patients.

Dr Gulati. I appreciate your comments. Unfortunately, I do not have great images here to show you regarding that. I can tell you that in at least in a couple of cases there appeared to be compression on the preoperative echocardiogram, but in the follow-up echocardiogram there did not appear to be any compression.

Dr Charles D. Fraser, Jr (Houston, Tex). I want to ask you for recommendations about a situation that you are not likely to always be involved in: What would you recommend to these patients after repair? If I understood you correctly, 4 patients had the translocation procedure, and 2 of them were asymptomatic. Presumably, ischemic testing before surgery was nonrevealing, as it has been in our experience sometimes. Then you do the operation and the patient is still asymptomatic. The next question is, what can they do? Can they participate in sports? Do we need to quit worrying about them? As Dr. Spray has mentioned, it is a little hard to envision how this is changing the natural history of this problem.

What are your recommendations? We argue about this bitterly sometimes with our cardiologists. Certainly the parents want to know: Okay, now you've done the operation. What can my child do?

Dr Gulati. That is a really difficult question. It is very hard to know exactly what the right answer is.

Our recommendation has been to avoid severely competitive sports. We have done stress testing on some patients to see whether it would be safe to go back to sports, but there are definitely patients in the autopsy series who had normal stress tests but died from the lesion. Thus it is very hard to know the right thing to recommend. 
Despite our recommendations, we have had several patients who have insisted on returning to competitive sports. One patient, who had a reimplantation, is now running $800-\mathrm{m}$ to $1600-\mathrm{m}$ races and winning them. We had initially recommended that he not go back to sports, but he is doing great. Fortunately, that patient had been symptomatic, so we had a gauge as to whether we had done anything. In asymptomatic patients, it is really hard to know whether the risk of sudden death has been eliminated, so it is difficult to make definitive recommendations. The only thing that will tell is the long-term studies, and we will be monitoring these patients long term.

\section{New Editorial Office}

Effective January 1, 2008, Lawrence H. Cohn, MD will become editor of The Journal of Thoracic and Cardiovascular Surgery. As of that date, please direct all queries to:

Ryan Walther, Managing Editor

Journal of Thoracic and Cardiovascular Surgery

American Association for Thoracic Surgery

900 Cummings Center, Suite 221-U

Beverly, MA 01915

Telephone: 978-299-4505

Fax: 978-524-8330

E-mail: jtcvs@aats.org

Continue to submit all manuscripts to Editorial Manager at http://www.editorialmanager.com/jtcvs. 

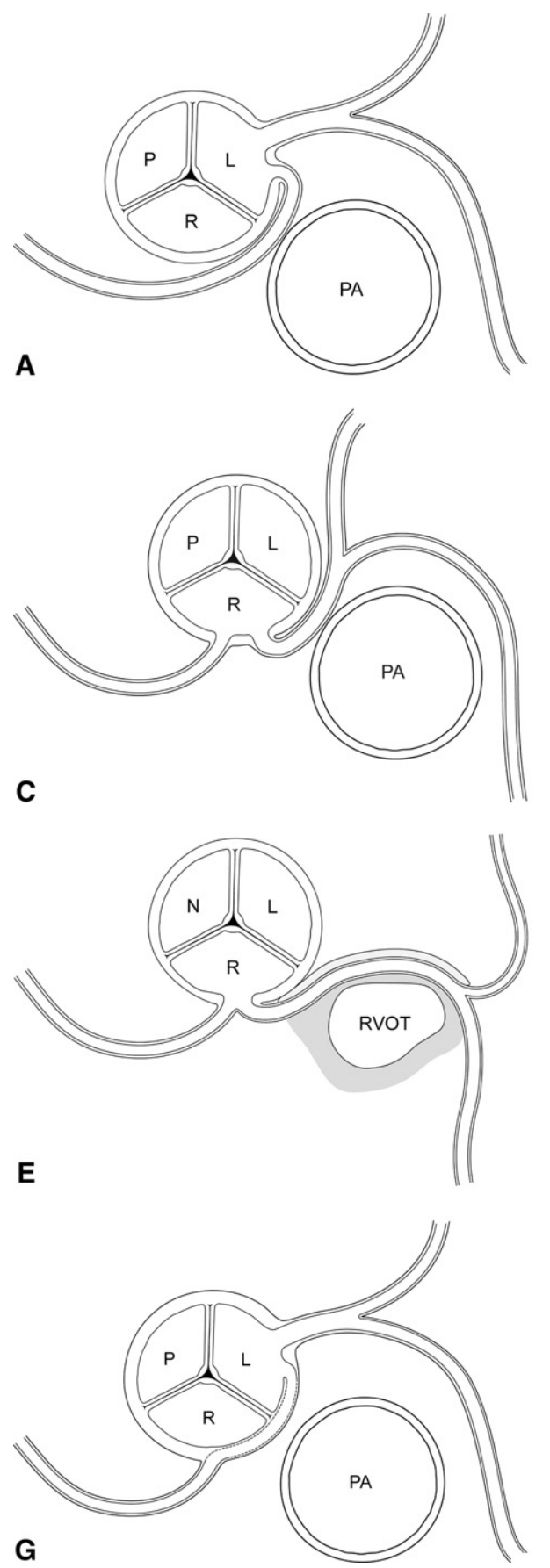
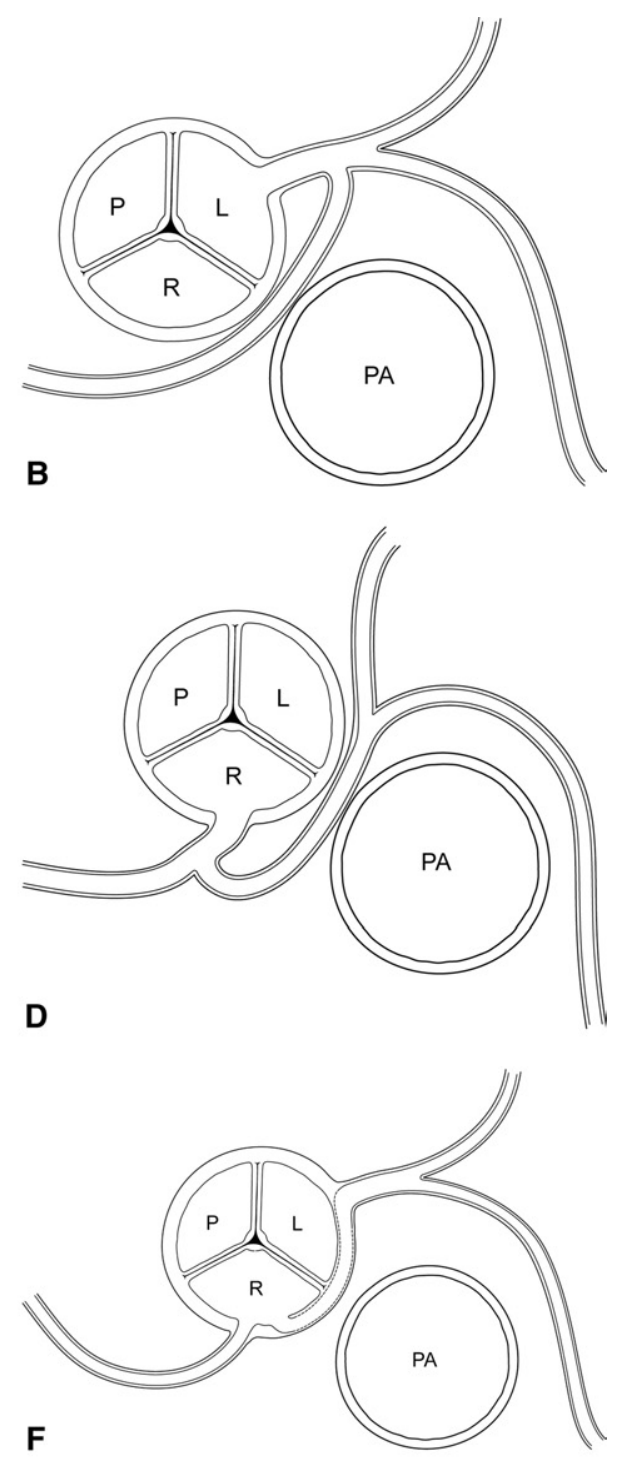

$\mathbf{F}$
Figure E1. High-risk anomalous coronary artery patterns. PA, Pulmonary artery; $\boldsymbol{P}$, posterior "noncoronary" sinus of Valsalva; $L$, left sinus of Valsalva; $R$, right sinus of Valsalva. A, Anomalous right coronary artery (RCA) with separate ostium, arising from left coronary sinus (LCS), coursing interarterially, between the PA and aorta, without intramural course ( $n=2$ in this series). B, Anomalous RCA with single ostium (shared with left coronary artery [LCA]), arising from LCS, and coursing interarterially between the PA and aorta, without intramural course ( $n=1)$. C, Anomalous LCA with separate ostium, arising from right coronary sinus (RCS), coursing interarterially between the PA and aorta, without intramural course $(\mathbf{n}=\mathbf{0})$. D, Anomalous LCA with single ostium (shared with RCA), arising from right coronary sinus (RCS), and coursing interarterially between the PA and aorta, without intramural course ( $n=2$ ). $E$, Anomalous LCA with single ostium (shared with RCA), arising from the RCS and coursing interarterially and intramuscularly between the PA and aorta, without intramural course $(n=1)$ RVOT, Right ventricular outflow tract. F, Anomalous LCA with separate ostium, arising from the RCS, coursing interarterially and between the PA and aorta, with intramural course ( $N$ $=4$ ). G, Anomalous RCA with separate ostium, arising from the, coursing interarterially between the PA and aorta, with intramural course (n $=7$ ). Although not illustrated here, anomalous RCA with common ostium (shared with LCA) arising from the LCS, coursing interarterially between the PA and aorta, with intramural course, may occur $(n=1)$. 


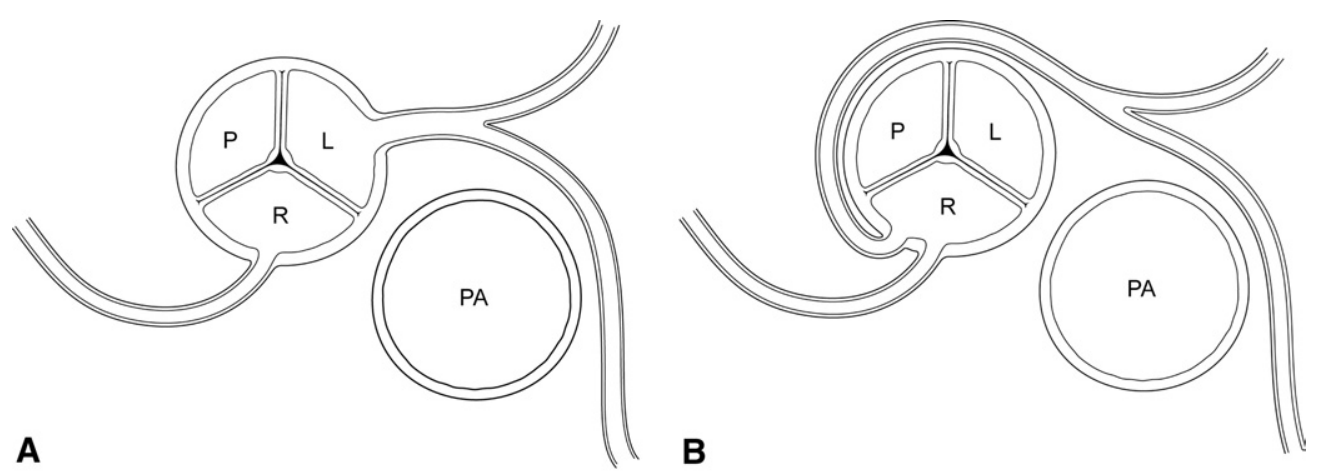

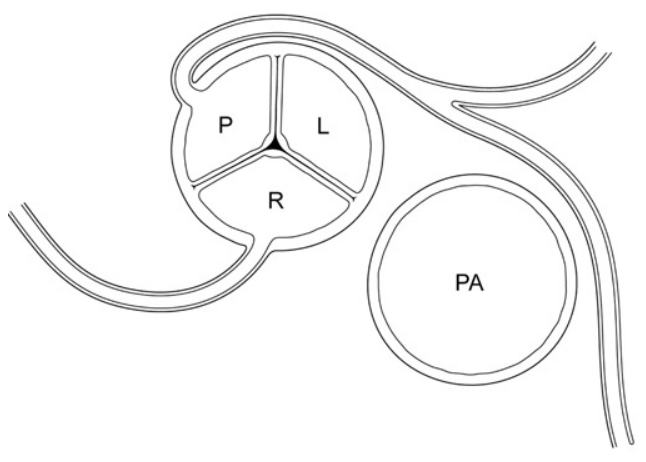

C

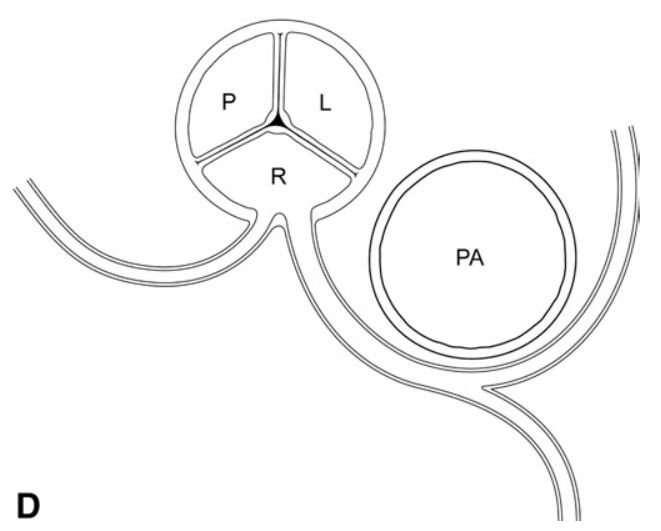

Figure E2. Normal and low-risk anomalous coronary artery. B through D are considered low risk if there is no abnormality of the coronary origin. A, Normal coronary arteries. B, Anomalous LCA with separate ostium, arising from RCS, coursing posteriorly, around aorta. C, Anomalous LCA with separate ostium, arising from posterior (noncoronary) sinus, coursing posteriorly, around aorta. D, Anomalous LCA with separate ostium, arising from RCS, coursing anteriorly, around PA. For abbreviations, see Figures E1 and E2. 

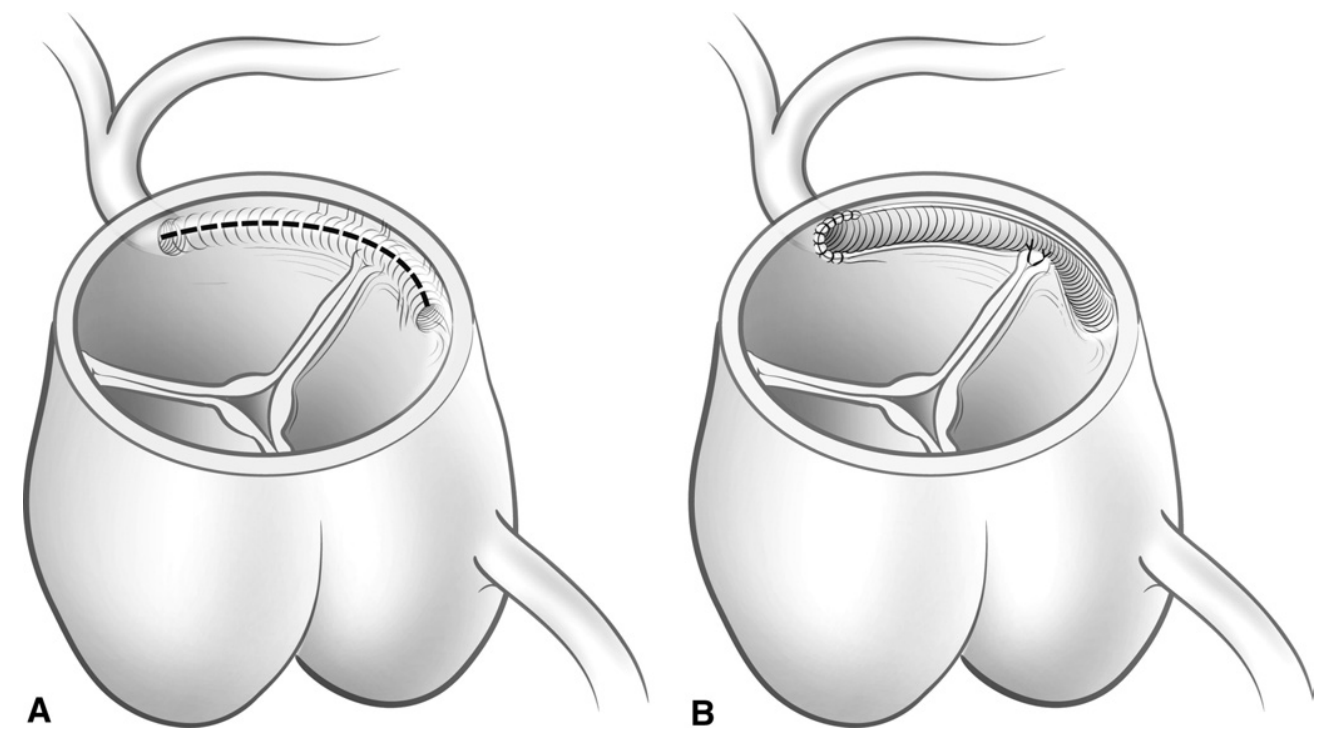

Figure E3. Unroofing. Patient with anomalous LCA from LCS, with intramural course. A, The intramural course is opened up over its full length, along the dotted line. B, The edges are trimmed. If necessary, the cut edges are reinforced with fine suture and the aortic valve commissure is resuspended. This eliminates the intramural and interarterial course and creates a neo-ostium without angulation or slit-like opening. For abbreviations, see Figures E1 and E2. 


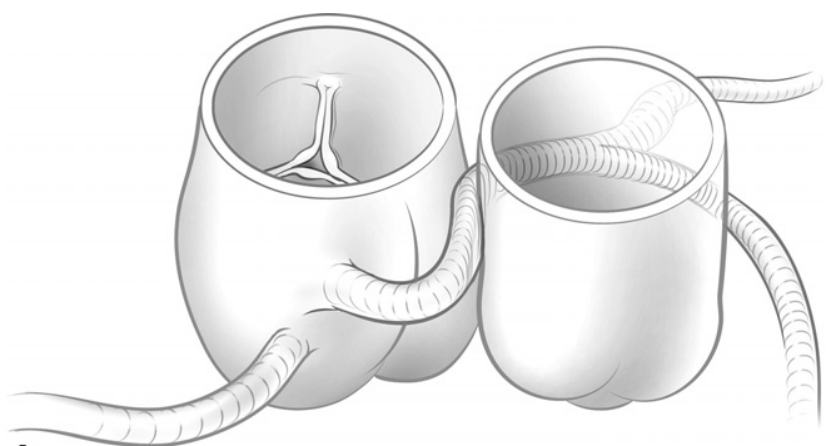

A

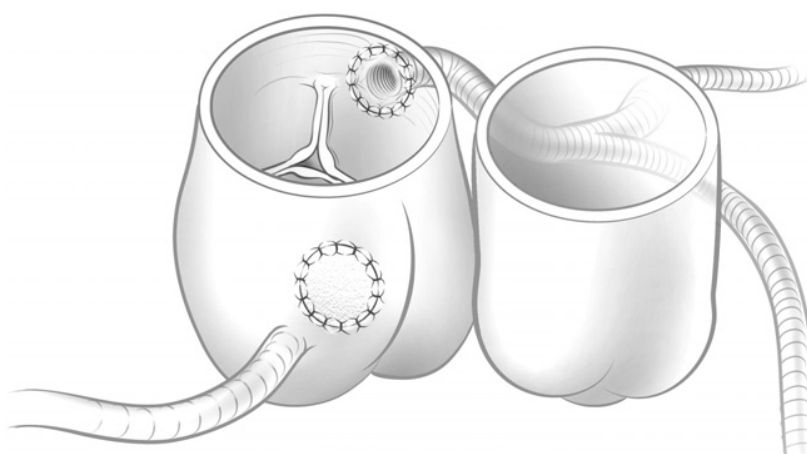

B

Figure E4. Coronary artery reimplantation. Anomalous LCA arising from the RCS. A, Before repair. B, Coronary artery is detached with button of aortic tissue and reimplanted above correct sinus. Placement is slightly superior to avoid kinking. For abbreviations, see Figures E1 and E2.
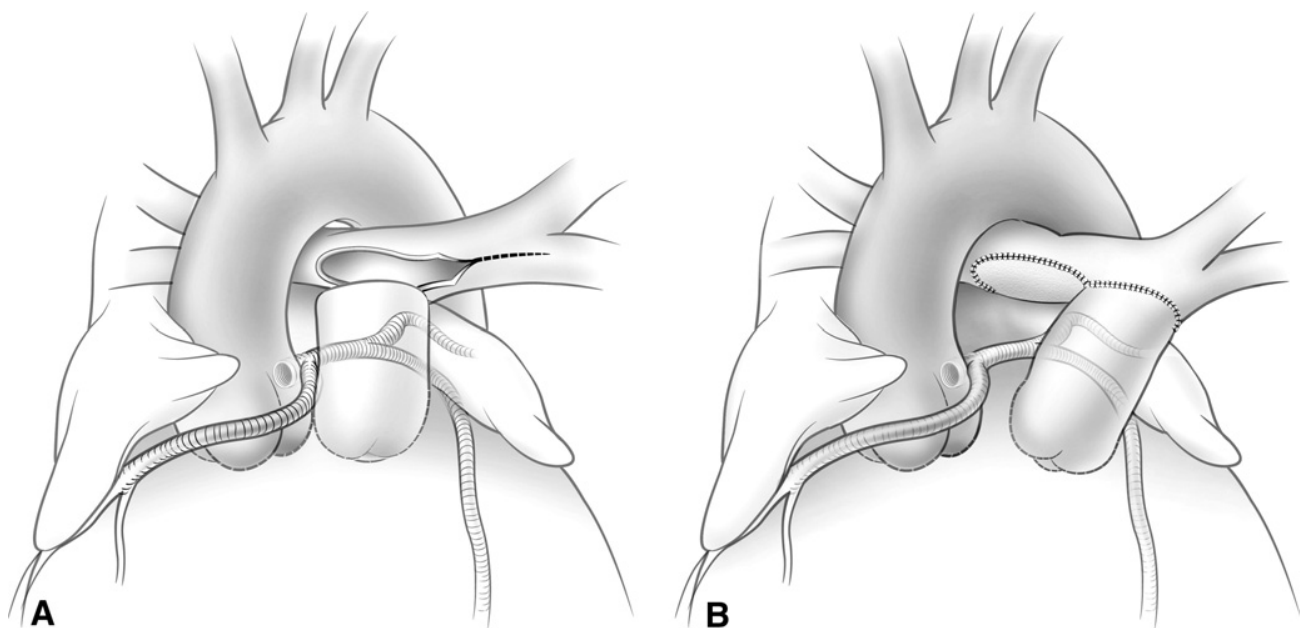

Figure E5. Lateral PA translocation. Anomalous RCA from the LCS with single ostium and normal proximal course. A, Main PA is transected at the bifurcation and left branch PA is opened along line. B, Main PA is translocated and reimplanted on the left PA, and original site is closed with pericardial patch. This moves the main PA laterally, toward the left, and reduces possible compression of anomalous artery. 

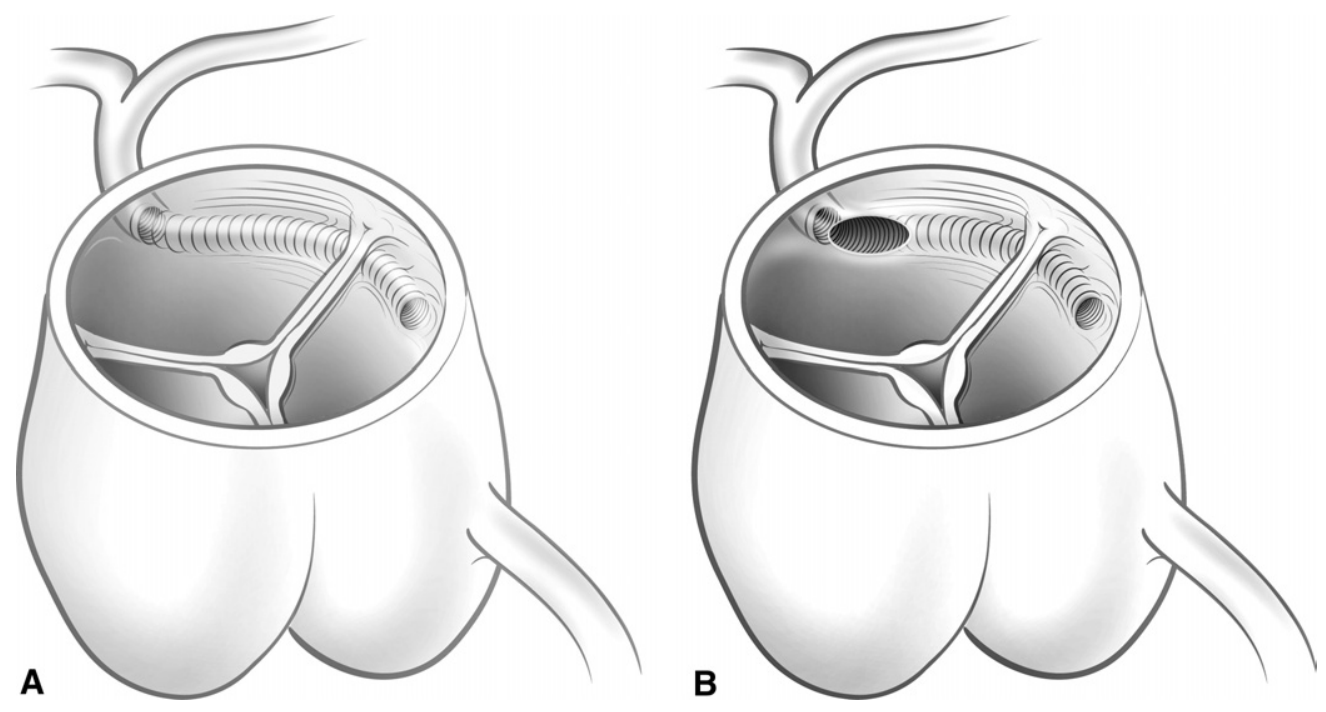

Figure E6. "Fenestration"/limited unroofing. A, Anomalous left main coronary artery arising from the wrong coronary sinus with low intramural course, below sinotubular junction. B, Fenestration: a limited unroofing is performed only in the correct coronary sinus, creating a neo-ostium without angulation, in the correct coronary sinus, and eliminating the interarterial course, without disturbing the aortic valve commissure. 